\title{
GAUGe Symmetry AND SUPERSYMMETRY OF MULTIPLE M2-BRANES
}

\author{
JONATHAN BAGGER ${ }^{1}$ \\ Department of Physics and Astronomy \\ Johns Hopkins University \\ 3400 North Charles Street \\ Baltimore, MD 21218, USA \\ and \\ NEIL LAMBERT ${ }^{2}$ \\ Department of Mathematics \\ King's College London \\ The Strand \\ London WC2R 2LS, UK
}

\begin{abstract}
In previous work we proposed a field theory model for multiple M2-branes based on an algebra with a totally antisymmetric triple product. In this paper we gauge a symmetry that arises from the algebra's triple product. We then construct a supersymmetric theory that is consistent with all the symmetries expected of a multiple M2-brane theory: 16 supersymmetries, conformal invariance, and an $S O(8)$ R-symmetry that acts on the eight transverse scalars. The gauge field is not dynamical. The result is a new type of maximally supersymmetric gauge theory in three dimensions.
\end{abstract}

\footnotetext{
${ }^{1}$ bagger@jhu.edu

${ }^{2}$ neil.lambert@kcl.ac.uk
} 


\section{Introduction}

The branes of M-theory are important but still very much mysterious objects. While the dynamics of a single M-brane is well understood, very little is known about the interactions of multiple M-branes. For a current review of M-branes and their interactions, see [1].

In a recent paper [2], we proposed a model of multiple M2-branes based on an algebra that admits a totally antisymmetric triple product. (The triple product can be constructed, for example, from the associator in a nonassociative algebra.) Examination of the supersymmetry algebra suggested that the theory has a local gauge symmetry that arises from the triple product.

In ref. [2] the nature of these gauge transformations was not clear, so the model presented contained just the scalar and fermi fields. Moreover, it was invariant under just four supersymmetries. In this paper we will study the gauge symmetry in more detail. We will show how to gauge the local symmetry and obtain a conformal and gauge-invariant action with all 16 supersymmetries. The theory an $S O(8)$ R-symmetry that acts on the eight transverse scalars, a nonpropagating gauge field, and no free parameters, modulo a rescaling of the structure constants. The gauge field ensures that the supersymmetry algebra closes (up to a gauge transformation) on shell.

Apart from our motivation to obtain a worldvolume theory for multiple M2-branes, it is generally worthwhile to to pursue extensions to YangMills gauge theory and to explore the possible relevance of nonassociative structures to theoretical physics and geometry. In fact, beyond the model proposed in [2], there are other physical examples [3]-[8] in which fuzzy threespheres arise. Such objects presumably require an algebraic structure that is based on an antisymmetric triple product, so the results discussed here may be relevant.

The rest of this paper is organized as follows. In section two we present the details of the algebraic structure that we require and show how it leads to a natural symmetry. In section three we gauge the symmetry by introducing a vector gauge field. In section four we construct a gauge-invariant supersymmetric theory with 16 supercharges acting on the scalars, vector and fermions. The superalgebra closes on a set of equations of motion that are invariant under supersymmetry. We show that the equations of motion arise from a supersymmetric action consistent with all the known continuous symmetries of the M2-brane. Section five contains some closing comments.

We also include two appendices. The first provides a concrete example 
of a three-algebra; the second lists some Clifford algebra identities that are relevant to the computations in section four.

While this paper was is in preparation, we received ref. [9], in which the algebraic structures underlying multiple M2-branes are discussed. Furthermore, in a revised version (v4), the gauged supersymmetry algebra was found to close using the fermion and vector equations of motion. The fields are elements of a Lie algebra constructed out of the semidirect product of two other algebras, one of which has a triple product. The superalgebra presented in [9] looks similar to ours. It would be interesting to see if the two algebraic structures are, in fact, the same.

\section{Some Algebraic Details}

The model presented in [2] was based on a nonassociative algebra. In algebra one commonly introduces the associator

$$
<A, B, C>=(A \cdot B) \cdot C-A \cdot(B \cdot C)
$$

which vanishes in an associative algebra. In what follows we need the antisymmeterized associator

$$
\begin{aligned}
{[A, B, C]=} & <A, B, C>+<B, C, A>+<C, A, B> \\
& -<A, C, B>-<B, A, C>-<C, B, A>,
\end{aligned}
$$

which is what one finds by expanding out the Jacobi identity $[[A, B], C]+$ $[[B, C], A]+[[C, A], B]$. In a nonassociative algebra, the antisymmeterized associator leads to a natural triple product structure.

To define an action we require a trace-form on the algebra $\mathcal{A}$. This is a bilinear map $\operatorname{Tr}: \mathcal{A} \times \mathcal{A} \rightarrow \mathbb{C}$ that is symmetric and invariant:

$$
\operatorname{Tr}(A, B)=\operatorname{Tr}(B, A) \quad \operatorname{Tr}(A \cdot B, C)=\operatorname{Tr}(A, B \cdot C) .
$$

We also assume 'Hermitian' conjugation \# and positivity, which implies $\operatorname{Tr}\left(A^{\#}, A\right) \geq 0$ for any $A \in \mathcal{A}$ (with equality if and only if $A=0$ ). The invariance property implies that

$$
\begin{aligned}
\operatorname{Tr}(<A, B, C>, D) & =\operatorname{Tr}((A \cdot B) \cdot C, D)-\operatorname{Tr}(A \cdot(B \cdot C), D) \\
& =\operatorname{Tr}(A \cdot B, C \cdot D)-\operatorname{Tr}(A,(B \cdot C) \cdot D) \\
& =-\operatorname{Tr}(A,<B, C, D>) .
\end{aligned}
$$


It also follows that

$$
\operatorname{Tr}([A, B, C], D)=-\operatorname{Tr}(A,[B, C, D]) .
$$

More generally we only require that the algebra admit a totally antisymmetric trilinear product $[\cdot, \cdot, \cdot]$ that satisfies (5). In particular, the antisymmetric product need not arise from a non-associative product on the algebra. We call such an algebra a three-algebra. Note that a three-algebra need not contain a bilinear product and hence is not necessarily an algebra in the usual sense.

In [2] we found that closure of the 16 component supersymmetry algebra leads to the variation

$$
\delta X^{I} \propto i \bar{\epsilon}_{2} \Gamma_{J K} \epsilon_{1}\left[X^{J}, X^{K}, X^{I}\right]
$$

which can be viewed as a local version of the global symmetry transformation

$$
\delta X=[\alpha, \beta, X]
$$

where $\alpha, \beta \in \mathcal{A}$. For (7) to be a symmetry, it must act as a derivation,

$$
\delta([X, Y, Z])=[\delta X, Y, Z]+[X, \delta Y, Z]+[X, Y, \delta Z] .
$$

This leads to the 'fundamental' identity (which has also appeared in [10]-[12])

$$
[\alpha, \beta,[X, Y, Z]]=[[\alpha, \beta, X], Y, Z]+[X,[\alpha, \beta, Y], Z]+[X, Y,[\alpha, \beta, Z]] .
$$

We proceed assuming that this identity holds. It will play a role analogous to the Jacobi identity in ordinary Lie algebra, where it arises from demanding that the transformation $\delta X=[\alpha, X]$ act as a derivation.

It is convenient to introduce a basis $T^{a}$ for the algebra $\mathcal{A}$. On physical grounds we assume that all the generators are Hermitian, in the sense that $\left(T^{a}\right)^{\#}=T^{a}$. We then expand the field $X=X_{a} T^{a}, a=1, \ldots, N$, where $N$ is the dimension of $\mathcal{A}$ (and not the number of M2-branes). We introduce the 'structure' constants

$$
\left[T^{a}, T^{b}, T^{c}\right]=f_{d}^{a b c} T^{d},
$$

from which is it is clear that $f_{d}^{a b c}=f^{[a b c]}$. The trace-form provides a metric

$$
h^{a b}=\operatorname{Tr}\left(T^{a}, T^{b}\right)
$$


that we can use to raise indices: $f^{a b c d}=h^{d e} f^{a b c}$. Again on physical grounds we assume that $h^{a b}$ is positive definite. The condition (5) on the trace-form implies that

$$
f^{a b c d}=-f^{d b c a}
$$

and this further implies that $f^{a b c d}=f^{[a b c d]}$, in analogy with the familiar result in Lie algebras. In a basis form the fundamental identity (9) becomes

$$
f_{d}^{e f g} f_{g}^{a b c}=f_{g}^{e f a} f_{d}^{b c g}+f_{g}^{e f b} f_{d}^{c a g}+f_{g}^{e f c} f_{d}^{a b g} .
$$

We can augment this algebra by including an element $T^{0}$ that associates with everything, or more precisely, that satisfies $f_{d}^{0 a b}=0$. If we assume that $h^{0 b}=0$ if $b \neq 0$, we find $f_{0}^{a b c}=0$. Thus this mode decouples and it can be interpreted as the centre-of-mass coordinate.

The symmetry transformation (7) can be written as

$$
\delta X_{d}=f_{d}^{a b c} \alpha_{a} \beta_{b} X_{c} .
$$

However the notation allows for the more general transformation

$$
\delta X_{d}=f^{a b c}{ }_{d} \Lambda_{a b} X_{c},
$$

which we assume from now on. In particular, the transformation (6) corresponds to the choice

$$
\Lambda_{a b} \propto i \bar{\epsilon}_{1} \Gamma_{J K} \epsilon_{2} X_{a}^{J} X_{b}^{K} .
$$

Note that the generator $\Lambda_{a b}$ cannot in general be written as $\alpha_{[a} \beta_{b]}$ for a single pair of vectors $\left(\alpha_{a}, \beta_{b}\right)$. However, $\Lambda_{a b}$ can always be written as a sum over $N$ such pairs.

To see that the action is invariant under global symmetries of this form, we observe that for any $Y$,

$$
\begin{aligned}
\frac{1}{2} \delta \operatorname{Tr}(Y, Y) & =\operatorname{Tr}(\delta Y, Y) \\
& =h^{d e} \delta Y_{d} Y_{e} \\
& =h^{d e} \Lambda_{a b} f_{d}^{a b c} Y_{c} Y_{e} \\
& =f^{a b c e} \Lambda_{a b} Y_{c} Y_{e} \\
& =0,
\end{aligned}
$$

by the antisymmetry of $f^{a b c e}$. In addition, the fundamental identity ensures that

$$
\left(\delta\left[X^{I}, X^{J}, X^{K}\right]\right)_{a}=f^{c d b}{ }_{a} \Lambda_{c d}\left[X^{I}, X^{J}, X^{K}\right]_{b}
$$


Thus the Lagrangian

$$
\mathcal{L}=-\frac{1}{2} \operatorname{Tr}\left(\partial_{\mu} X^{I}, \partial^{\mu} X^{I}\right)-3 \kappa^{2} \operatorname{Tr}\left(\left[X^{I}, X^{J}, X^{K}\right],\left[X^{I}, X^{J}, X^{K}\right]\right),
$$

is invariant under the symmetry $\delta X_{a}^{I}=f^{c d b}{ }_{a} \Lambda_{c d} X_{b}^{I}$.

\section{Gauging the Symmetry}

We now wish to promote the global symmetry discussed above to a local one. To that end we introduce a covariant derivative $D_{\mu} X$ such that $\delta\left(D_{\mu} X\right)=$ $D_{\mu}(\delta X)+\left(\delta D_{\mu}\right) X$. If we let

$$
\delta X_{a}=\Lambda_{c d} f^{c d b}{ }_{a} X_{b} \equiv \tilde{\Lambda}_{a}^{b} X_{b},
$$

then the natural choice is to take

$$
\left(D_{\mu} X\right)_{a}=\partial_{\mu} X_{a}-\tilde{A}_{\mu}^{b}{ }_{a}^{b} X_{b}
$$

where $\tilde{A}_{\mu}{ }^{b} a \equiv f^{c d b}{ }_{a} A_{\mu c d}$ is a gauge field with two algebraic indices. We can therefore think of $\tilde{A}_{\mu}^{b}{ }_{a}^{b}$ as living in the space of linear maps from $\mathcal{A}$ to itself, in analogy with the adjoint representation of a Lie algebra. The field $X$ is then, in some sense, in the fundamental representation. The gauge field acts as an element of $g l(N)$, where $N$ is the dimension of $\mathcal{A}$. Furthermore, as a consequence of the antisymmetry of $f^{a b c d}$, the symmetry algebra is contained in $s o(N)$.

A little calculation shows that the covariant derivative is obtained by taking

$$
\begin{aligned}
\delta \tilde{A}_{\mu a}{ }^{b} a & =\partial_{\mu} \tilde{\Lambda}_{a}^{b}-\tilde{\Lambda}^{b}{ }_{c} \tilde{A}_{\mu{ }^{c}{ }_{a}}+\tilde{A}_{\mu}{ }^{b}{ }_{c} \tilde{\Lambda}^{c}{ }_{a} \\
& \equiv D_{\mu} \tilde{\Lambda}^{b}{ }_{a} .
\end{aligned}
$$

Indeed, this is the usual form of a gauge transformation. The field strength is defined as

$$
\left(\left[D_{\mu}, D_{\nu}\right] X\right)_{a}=\tilde{F}_{\mu \nu}{ }^{b}{ }_{a} X_{b}
$$

which leads to

$$
\tilde{F}_{\mu \nu}{ }^{b} a=\partial_{\nu} \tilde{A}_{\mu}{ }^{b}{ }_{a}-\partial_{\mu} \tilde{A}_{\nu}{ }^{b}{ }_{a}-\tilde{A}_{\mu}{ }^{b}{ }_{c} \tilde{A}_{\nu}{ }^{c}{ }_{a}+\tilde{A}_{\nu}{ }^{b}{ }_{c} \tilde{A}_{\mu}{ }^{c}{ }^{c} .
$$


The resulting Bianchi identity is $D_{[\mu} \tilde{F}_{\nu \lambda]^{b}}^{b}=0$. One also finds that

$$
\delta \tilde{F}_{\mu \nu a}^{b}=-\tilde{\Lambda}_{c}^{b} \tilde{F}_{\mu \nu}^{c}{ }_{a}+\tilde{F}_{\mu \nu}{ }_{c}^{b} \tilde{\Lambda}_{a}^{c} \text {. }
$$

These expressions are identical to what one finds in an ordinary gauge theory based on a Lie algebra, where the gauge field is in the adjoint representation. Here the gauge field takes values in the space of linear maps of $\mathcal{A}$ into itself. The triple product allows one to construct linear maps on $\mathcal{A}$ from two elements of $\mathcal{A}$.

In particular consider the set $\mathcal{G}$ of all $N \times N$ matrices $\tilde{\Lambda}_{a}^{b}=\Lambda_{c d} f^{c d b}{ }_{a}$, where $\Lambda_{c d}$ is arbitrary. The fundamental identity ensures that this set is closed under the ordinary matrix commutator. Thus $\mathcal{G}$ defines a matrix Lie algebra that is a subalgebra of $s o(N)$. The fundamental identity implies that $f^{a b c d}$ is an invariant 4 -form of $\mathcal{G}$. Thus every three-algebra generates a Lie algebra with an invariant 4-form. However, it is unclear whether or not the existence of an invariant 4-form in a Lie algebra is sufficient to ensure that its fundamental representation is a three-algebra that satisfies the fundamental identity.

\section{Supersymmetrizing the Gauged Theory}

We now show how to supersymmeterize the gauged multi-M2-brane model in a manner consistent will all the continuous symmetries expected of a multiple M2-brane theory, namely 16 supersymmetries, conformal invariance, and an $S O(8)$ R-symmetry that acts on the eight transverse scalars. We first recall the structure of the full superalgebra with 16-component spinors. In [2] we argued that the general form is

$$
\begin{aligned}
\delta X^{I} & =i \bar{\epsilon} \Gamma^{I} \Psi \\
\delta \Psi & =\partial_{\mu} X^{I} \Gamma^{\mu} \Gamma^{I} \epsilon+\kappa\left[X^{I}, X^{J}, X^{K}\right] \Gamma^{I J K} \epsilon,
\end{aligned}
$$

where $\kappa$ is a constant. We then showed that this algebra does not close. However, closure on the scalars $X^{I}$ leads to the local symmetry

$$
\delta X^{I} \propto i \bar{\epsilon}_{2} \Gamma_{J K} \epsilon_{1}\left[X^{J}, X^{K}, X^{I}\right]
$$

that we gauged above. 
Let us apply the ideas of the previous section to gauge this symmetry. We start by introducing the gauge field $\tilde{A}_{\mu}{ }^{b}{ }_{a}$ with its associated covariant derivative. The supersymmetry transformations then take the form

$$
\begin{aligned}
\delta X_{a}^{I} & =i \bar{\epsilon} \Gamma^{I} \Psi_{a} \\
\delta \Psi_{a} & =D_{\mu} X_{a}^{I} \Gamma^{\mu} \Gamma^{I} \epsilon+\kappa X_{b}^{I} X_{c}^{J} X_{d}^{K} f_{a}^{b c d} \Gamma^{I J K} \epsilon \\
\delta \tilde{A}_{\mu}{ }^{b} a & =i \bar{\epsilon} \Gamma_{\mu} \Gamma_{I} X_{c}^{I} \Psi_{d} f^{c d b}{ }_{a} .
\end{aligned}
$$

(A similar, possibly identical, form for the gauge field variation was used in [9].)

This algebra can be made to close on shell. We first consider the scalars. We find that the transformations close into a translation and a gauge transformation;

$$
\left[\delta_{1}, \delta_{2}\right] X_{a}^{I}=v^{\mu} D_{\mu} X_{a}^{I}+\tilde{\Lambda}^{b}{ }_{a} X_{b}^{I}
$$

where

$$
v^{\mu}=-2 i \bar{\epsilon}_{2} \Gamma^{\mu} \epsilon_{1}, \quad \tilde{\Lambda}_{a}^{b}=6 i \kappa \bar{\epsilon}_{2} \Gamma_{J K} \epsilon_{1} X_{c}^{J} X_{d}^{K} f_{a}^{c d b} .
$$

We then consider the fermions. Evaluating $\left[\delta_{1}, \delta_{2}\right] \Psi_{a}$, we find two separate terms involving $\bar{\epsilon}_{2} \Gamma_{\mu} \Gamma_{I J K L} \epsilon_{1}$ that must cancel for closure. This implies

$$
\kappa=-1 / 6,
$$

so there is no free parameter. Proceeding further we compute

$$
\begin{aligned}
{\left[\delta_{1}, \delta_{2}\right] \Psi_{a}=} & v^{\mu} D_{\mu} \Psi_{a}+\tilde{\Lambda}_{a}^{b} \Psi_{b} \\
& +i\left(\bar{\epsilon}_{2} \Gamma_{\nu} \epsilon_{1}\right) \Gamma^{\nu}\left(\Gamma^{\mu} D_{\mu} \Psi_{a}+\frac{1}{2} \Gamma_{I J} X_{c}^{I} X_{d}^{J} \Psi_{b} f^{c d b}{ }_{a}\right) \\
& -\frac{i}{4}\left(\bar{\epsilon}_{2} \Gamma_{K L} \epsilon_{1}\right) \Gamma^{K L}\left(\Gamma^{\mu} D_{\mu} \Psi_{a}+\frac{1}{2} \Gamma_{I J} X_{c}^{I} X_{d}^{J} \Psi_{b} f^{c d b}{ }_{a}\right) .
\end{aligned}
$$

Closure requires that the second and third lines vanish. This determines the fermionic equation of motion;

$$
\Gamma^{\mu} D_{\mu} \Psi_{a}+\frac{1}{2} \Gamma_{I J} X_{c}^{I} X_{d}^{J} \Psi_{b} f_{a}^{c d b}=0 .
$$

Thus on shell we see that

$$
\left[\delta_{1}, \delta_{2}\right] \Psi_{a}=v^{\mu} D_{\mu} \Psi_{a}+\tilde{\Lambda}_{a}^{b} \Psi_{b},
$$


as required.

We finally turn to $\left[\delta_{1}, \delta_{2}\right] \tilde{A}_{\mu}{ }^{b}{ }_{a}$. Here we again find a term involving $\bar{\epsilon}_{2} \Gamma_{\mu} \Gamma_{I J K L} \epsilon_{1}$ :

$$
-\frac{i}{3}\left(\bar{\epsilon}_{2} \Gamma_{\mu} \Gamma_{I J K L} \epsilon_{1}\right) X_{c}^{I} X_{e}^{J} X_{f}^{K} X_{g}^{L} f_{d f}^{e f g} f^{c d b}{ }_{a} .
$$

Happily this term vanishes as a consequence of the fundamental identity. Continuing, we find

$$
\begin{aligned}
{\left[\delta_{1}, \delta_{2}\right] \tilde{A}_{\mu a}{ }^{b}=} & 2 i\left(\bar{\epsilon}_{2} \Gamma^{\nu} \epsilon_{1}\right) \epsilon_{\mu \nu \lambda}\left(X_{c}^{I} D^{\lambda} X_{d}^{I}+\frac{i}{2} \bar{\Psi}_{c} \Gamma^{\lambda} \Psi_{d}\right) f^{c d b}{ }_{a} \\
& -2 i\left(\epsilon_{2} \Gamma_{I J} \epsilon_{1}\right) X_{c}^{I} D_{\mu} X_{d}^{J} f^{c d b}{ }_{a} .
\end{aligned}
$$

To close the algebra we fix the $\tilde{A}_{\mu}^{b}{ }_{a}$ equation of motion;

$$
\tilde{F}_{\mu \nu a}{ }^{b}+\epsilon_{\mu \nu \lambda}\left(X_{c}^{J} D^{\lambda} X_{d}^{J}+\frac{i}{2} \bar{\Psi}_{c} \Gamma^{\lambda} \Psi_{d}\right) f^{c d b}{ }_{a}=0,
$$

so that on shell,

$$
\left[\delta_{1}, \delta_{2}\right] \tilde{A}_{\mu a}^{b}=v^{\nu} \tilde{F}_{\mu \nu a}^{b}+D_{\mu} \tilde{\Lambda}_{a}^{b} .
$$

Note that $\tilde{A}_{\mu d}^{c}$ contains no local degrees of freedom, as required. We see that the 16 supersymmetries close on shell.

To find the bosonic equations of motion, we take the supervariation of the fermion equation of motion. This gives

$$
\begin{aligned}
0= & \Gamma^{I}\left(D^{2} X_{a}^{I}-\frac{i}{2} \bar{\Psi}_{c} \Gamma^{I J} X_{d}^{J} \Psi_{b} f_{a}^{c d b}+\frac{1}{2} f_{a}^{b c d} f_{d}^{e f g} X_{b}^{J} X_{c}^{K} X_{e}^{I} X_{f}^{J} X_{g}^{K}\right) \epsilon \\
& +\Gamma^{I} \Gamma_{\lambda} X_{b}^{I}\left(\frac{1}{2} \varepsilon^{\mu \nu \lambda} \tilde{F}_{\mu \nu}{ }^{b}{ }_{a}-X_{c}^{J} D^{\lambda} X_{d}^{J} f^{c d b}{ }_{a}-\frac{i}{2} \bar{\Psi}_{c} \Gamma^{\lambda} \Psi_{d} f^{c d b}{ }_{a}\right) \epsilon .
\end{aligned}
$$

The second term vanishes as a consequence of the vector equation of motion (37). The first term determines the scalar equations of motion,

$$
D^{2} X_{a}^{I}-\frac{i}{2} \bar{\Psi}_{c} \Gamma^{I J} X_{d}^{J} \Psi_{b} f_{a}^{c d b}-\frac{\partial V}{\partial X^{I a}}=0 .
$$

The potential is

$$
\begin{aligned}
V & =\frac{1}{12} f^{a b c d} f_{d}^{e f g} X_{a}^{I} X_{b}^{J} X_{c}^{K} X_{e}^{I} X_{f}^{J} X_{g}^{K} \\
& =\frac{1}{2 \cdot 3 !} \operatorname{Tr}\left(\left[X^{I}, X^{J}, X^{K}\right],\left[X^{I}, X^{J}, X^{K}\right]\right) .
\end{aligned}
$$


Let us summarize our results. The supersymmetry transformations are

$$
\begin{aligned}
\delta X_{a}^{I} & =i \bar{\epsilon} \Gamma^{I} \Psi_{a} \\
\delta \Psi_{a} & =D_{\mu} X_{a}^{I} \Gamma^{\mu} \Gamma^{I} \epsilon-\frac{1}{6} X_{b}^{I} X_{c}^{J} X_{d}^{K} f_{a}^{b c d} \Gamma^{I J K} \epsilon \\
\delta \tilde{A}_{\mu}{ }^{b} a & =i \bar{\epsilon} \Gamma_{\mu} \Gamma_{I} X_{c}^{I} \Psi_{d} f^{c d b}{ }_{a} .
\end{aligned}
$$

These supersymmetries close into translations and gauge transformations,

$$
\begin{aligned}
{\left[\delta_{1}, \delta_{2}\right] X_{a}^{I} } & =v^{\mu} \partial_{\mu} X_{a}^{I}+\left(\tilde{\Lambda}^{b}{ }_{a}-v^{\nu} \tilde{A}_{\left.\nu{ }_{a}{ }^{b} X_{b}^{I}\right)}\right. \\
{\left[\delta_{1}, \delta_{2}\right] \Psi_{a} } & =v^{\mu} \partial_{\mu} \Psi_{a}+\left(\tilde{\Lambda}^{b}{ }_{a}-v^{\nu} \tilde{A}_{\nu}{ }^{b}{ }_{a} \Psi_{b}\right) \\
{\left[\delta_{1}, \delta_{2}\right] \tilde{A}_{\mu}{ }^{b}{ }_{a} } & =v^{\nu} \partial_{\nu} \tilde{A}_{\mu}{ }^{b}{ }_{a}+\tilde{D}_{\mu}\left(\tilde{\Lambda}^{b}{ }_{a}-v^{\nu} \tilde{A}_{\nu}{ }^{b}{ }_{a}\right)
\end{aligned}
$$

after using the equations of motion

$$
\begin{aligned}
\Gamma^{\mu} D_{\mu} \Psi_{a}+\frac{1}{2} \Gamma_{I J} X_{c}^{I} X_{d}^{J} \Psi_{b} f^{c d b}{ }_{a} & =0 \\
D^{2} X_{a}^{I}-\frac{i}{2} \bar{\Psi}_{c} \Gamma^{I}{ }_{J} X_{d}^{J} \Psi_{b} f^{c d b}{ }_{a}-\frac{\partial V}{\partial X^{I a}} & =0 \\
\tilde{F}_{\mu \nu}{ }^{b}{ }_{a}+\varepsilon_{\mu \nu \lambda}\left(X_{c}^{J} D^{\lambda} X_{d}^{J}+\frac{i}{2} \bar{\Psi}_{c} \Gamma^{\lambda} \Psi_{d}\right) f_{a}^{c d b} & =0 .
\end{aligned}
$$

We have explicitly demonstrated that the supersymmetry variation of the fermion equation of motion vanishes, and that the algebra closes on shell. It follows that all the equations of motion are invariant under supersymmetry. Furthermore one can check using the fundamental identity that the Bianchi identity $\epsilon^{\mu \nu \lambda} D_{\mu} \tilde{F}_{\nu \lambda{ }_{a}{ }^{b}}=0$ is satisfied.

We close this section by presenting an action for this system. The equations of motion can be obtained from the Lagrangian

$$
\begin{aligned}
\mathcal{L}= & -\frac{1}{2}\left(D_{\mu} X^{a I}\right)\left(D^{\mu} X_{a}^{I}\right)+\frac{i}{2} \bar{\Psi}^{a} \Gamma^{\mu} D_{\mu} \Psi_{a}+\frac{i}{4} \bar{\Psi}_{b} \Gamma_{I J} X_{c}^{I} X_{d}^{J} \Psi_{a} f^{a b c d} \\
& -V+\frac{1}{2} \varepsilon^{\mu \nu \lambda}\left(f^{a b c d} A_{\mu a b} \partial_{\nu} A_{\lambda c d}+\frac{2}{3} f^{c d a}{ }_{g} f^{e f g b} A_{\mu a b} A_{\nu c d} A_{\lambda e f}\right) .
\end{aligned}
$$

It is not hard to check that the action is gauge invariant and supersymmetric under the transformations (42). Note that (45) contains no free parameters, up to a rescaling of the structure constants. In fact, given the presence of the Chern-Simons term, it is natural to expect the $f^{a b c d}$ to be quantized [14]. 
It is important to note that the structure constants $f_{d}^{a b c}$ enter into the Chern-Simons term in a non-standard way. Viewed as a 3-form in an arbitrary dimension, this 'twisted' Chern-Simons term

$$
\Omega=\left(f^{a b c d} A_{\mu a b} \partial_{\nu} A_{\lambda c d}+\frac{2}{3} f^{c d a} f^{e f g b} A_{\mu a b} A_{\nu c d} A_{\lambda e f}\right) d x^{\mu} \wedge d x^{\nu} \wedge d x^{\lambda}
$$

satisfies

$$
d \Omega=F_{a b} \wedge \tilde{F}^{a b},
$$

where $\tilde{F}_{\mu \nu}{ }^{b}{ }_{a}=F_{\mu \nu c d} f^{c d b}{ }_{a}$. Also note that $\Omega$ is written in terms of $A_{\mu a b}$ and not the physical field $\tilde{A}_{\mu}{ }^{b} a=A_{\mu c d} f_{a}^{c d b}$ that appears in the supersymmetry transformations and equations of motion. However, one can check that $\Omega$ is invariant under shifts of $A_{\mu a b}$ that leave $\tilde{A}_{\mu}{ }^{b} a$ invariant. Thus it is locally well defined as a function of $\tilde{A}_{\mu a}^{b}$.

This theory provides an example of the type of model that was searched for in [13]. It is invariant under 16 supersymmetries and an $S O(8)$ Rsymmetry. It is also conformally invariant at the classical level. These are all the continuous symmetries that are expected of multiple M2-branes. Note that the Chern-Simons term naively breaks the parity that is expected to be a symmetry of the M2-brane worldvolume. However, we can make the Lagrangian parity invariant if we assign an odd parity to $f^{a b c d}$. In particular, if we invert $x^{2} \rightarrow-x^{2}$, we must then require that $X_{a}^{I}$ and $\tilde{A}_{\mu b}^{a}$ be parity even

for $\mu=0,1 ; \tilde{A}_{2 b}^{a}$ and $f^{a b c d}$ be parity odd; and $\Psi_{a} \rightarrow \Gamma_{2} \Psi_{a}$. Note that this assignment implies that $A_{\mu a b}$ is parity odd for $\mu=0,1$, while $A_{2 a b}$ is parity even.

\section{Conclusions}

In this paper we described the gauge symmetry that arises in the model of multiple M2-branes presented in [2]. We included a nonpropagating gauge field and obtained a theory that is invariant under all 16 supersymmetries with no free parameters, up to a rescaling. Thus the model presented in [2] can indeed be viewed as the truncation of a maximally supersymmetric theory to the scalar and fermion modes.

The Lagrangian given here is consistent with all the known symmetries of M2-branes. The M2-brane worldvolume theory is expected to arise as the strong coupling, conformal fixed point of a three-dimensional, maximally supersymmetric Yang-Mills gauge theory. Furthermore, in the large $N$ limit, 
it is conjectured to be dual to an $A d S_{4} \times S^{7}$ solution of M-theory. Thus the Lagrangian given here is a candidate for the strong coupling fixed point of a three-dimensional super Yang-Mills theory and a field theory dual of M-theory on $\mathrm{AdS}_{4} \times S^{7}$.

As mentioned in the introduction, similar results on the closure of the algebra have recently been reported in [9]. This paper adopts a different, but possibly equivalent, form for the algebra. We hope that these studies will warrant a deeper and fruitful investigation into the algebraic structure of multiple M2-branes as a step towards identifying the microscopic degrees of freedom of M-theory.

\section{Acknowledgements}

We would like to thank S. Cherkis and N. Cook for discussions. We also thank A. Gustavsson for alerting us to the revised version (v4) of [9]. JB is supported in part by the US National Science Foundation, grant NSFPHY-0401513. NL is supported in part by the PPARC grant PP/C507145/1 and the EU grant MRTN-CT-2004-512194 and would like to thank the Isaac Newton Institute where this work was completed.

\section{Appendix A: An Example}

In this appendix we provide an example of a three-algebra that satisfies the fundamental identity. The simplest nontrivial case corresponds to four generators, $a, b, \ldots=1,2,3,4$. If we normalize the generators such that $\operatorname{Tr}\left(T^{a}, T^{b}\right) \propto \delta^{a b}$, it then follows that

$$
f^{a b c d} \propto \varepsilon^{a b c d} .
$$

One can explicitly check that the fundamental identity is satisfied. In this case the space $\mathcal{G}$ generated by all matrices $\tilde{\Lambda}_{d}^{c}=\Lambda_{a b} f_{d}^{a b c}$ is the space of all $4 \times 4$ anti-symmetric matrices and hence $\mathcal{G}=s o(4)$ with the invariant 4 -form $\varepsilon^{a b c d}$.

It is also possible to realize this three-algebra as arising from a nonassociative algebra. In [2] we considered the three-algebra of Hermitian matrices that anti-commute with a fixed Hermitian matrix $G$, with $G^{2}=1$. We defined

$$
A \cdot B=Q A B Q
$$


where $Q=\frac{1}{\sqrt{2}}(1+i G)$. We also took $\operatorname{Tr}(A, B)=\operatorname{trace}\left(Q^{-1} A Q^{-1} B\right)$ where trace denotes the standard matrix trace. The associator turned out to be

$$
<A, B, C>=2 G A B C,
$$

and hence

$$
[A, B, C]=2 G(A B C \pm \text { cyclic }) .
$$

We also found that $G$ could play the role of translations as $[A, B, G]=0$ for all $A, B$ that anticommute with $G$.

Let us consider the case in which $\mathcal{A}$ has four generators, which we take to be the (Euclidean) four-dimensional $\gamma$-matrices with $G=\gamma_{5}$ and $Q=$ $\left(1+i \gamma_{5}\right) / \sqrt{2}$. The product is then $\gamma^{a} \cdot \gamma^{b}=Q \gamma^{a} \gamma^{b} Q=\gamma_{5} \gamma^{a} \gamma^{b}$, and one finds that

$$
<\gamma^{a}, \gamma^{b}, \gamma^{c}>=2 \gamma_{5} \gamma^{a} \gamma^{b} \gamma^{c}
$$

Thus

$$
\left[\gamma^{a}, \gamma^{b}, \gamma^{c}\right]=2 \cdot 3 ! \gamma_{5} \gamma^{a b c}=2 \cdot 3 ! \varepsilon^{a b c} \gamma^{d}
$$

and hence $f^{a b c d}=12 \varepsilon^{a b c d}$.

\section{Appendix B: Fierz and Other Identities}

In this appendix we present the Fierz identity that we use repeatedly above. All spinorial quantities are those of the eleven-dimensional Clifford algebra; we take them to be real. Let $\epsilon_{1}, \epsilon_{2}$ and $\chi$ be arbitrary spinors. The combination $\left(\bar{\epsilon}_{2} \chi\right) \epsilon_{1}-\left(\bar{\epsilon}_{1} \chi\right) \epsilon_{2}$ can then be written as

$$
\begin{aligned}
& \left(\bar{\epsilon}_{2} \chi\right) \epsilon_{1}-\left(\bar{\epsilon}_{1} \chi\right) \epsilon_{2}= \\
& -\frac{1}{16}\left(\left(\bar{\epsilon}_{2} \Gamma_{m} \epsilon_{1}\right) \Gamma^{m} \chi-\frac{1}{2 !}\left(\bar{\epsilon}_{2} \Gamma_{m n} \epsilon_{1}\right) \Gamma^{m n} \chi+\frac{1}{5 !}\left(\bar{\epsilon}_{2} \Gamma_{m n p q r} \epsilon_{1}\right) \Gamma^{m n p q r} \chi,\right.
\end{aligned}
$$

where $m, n, \ldots=0, \ldots, 10, \mu, \nu, \ldots=0,1,2$ and $I, J, \ldots=3,4, \ldots, 10$. If $\epsilon_{1}$ and $\epsilon_{2}$ have the same chirality with respect to $\Gamma_{012}$, then the only terms that contribute must have an even number of $I$ indices. Moreover, the expression is only nonvanishing when $\chi$ has the same $\Gamma_{012}$ chirality as $\epsilon_{1}$ and $\epsilon_{2}$. When this is the case, (54) reduces to

$$
\begin{aligned}
& \left(\bar{\epsilon}_{2} \chi\right) \epsilon_{1}-\left(\bar{\epsilon}_{1} \chi\right) \epsilon_{2}= \\
& -\frac{1}{16}\left(2\left(\bar{\epsilon}_{2} \Gamma_{\mu} \epsilon_{1}\right) \Gamma^{\mu} \chi-\left(\bar{\epsilon}_{2} \Gamma_{I J} \epsilon_{1}\right) \Gamma^{I J} \chi+\frac{1}{4 !}\left(\bar{\epsilon}_{2} \Gamma_{\mu} \Gamma_{I J K L} \epsilon_{1}\right) \Gamma^{\mu} \Gamma^{I J K L} \chi\right) .
\end{aligned}
$$


We also found the following identities useful:

$$
\begin{aligned}
\Gamma_{M} \Gamma^{I J} \Gamma^{M}= & 4 \Gamma^{I J} \\
\Gamma_{M} \Gamma^{I J K L} \Gamma^{M}= & 0 \\
\Gamma^{I J P} \Gamma^{K L M N} \Gamma_{P}= & -\Gamma^{I} \Gamma^{K L M N} \Gamma^{J}+\Gamma^{J} \Gamma^{K L M N} \Gamma^{I} \\
\Gamma^{I} \Gamma^{K L} \Gamma^{J}-\Gamma^{J} \Gamma^{K L} \Gamma^{I}= & 2 \Gamma^{K L} \Gamma^{I J}-2 \Gamma^{K J} \delta^{I L}+2 \Gamma^{K I} \delta^{J L}-2 \Gamma^{L I} \delta^{J K} \\
& +2 \Gamma^{L J} \delta^{I K}-4 \delta^{K J} \delta^{I L}+4 \delta^{K I} \delta^{J L} \\
\Gamma^{I J M} \Gamma^{K L} \Gamma_{M}= & 2 \Gamma^{K L} \Gamma^{I J}-6 \Gamma^{K J} \delta^{I L}+6 \Gamma^{K I} \delta^{J L}-6 \Gamma^{L I} \delta^{J K} \\
& +6 \Gamma^{L J} \delta^{I K}+4 \delta^{K J} \delta^{I L}-4 \delta^{K I} \delta^{J L} .
\end{aligned}
$$

\section{References}

[1] D. S. Berman, arXiv:0710.1707 [hep-th].

[2] J. Bagger and N. Lambert, arXiv:hep-th/0611108.

[3] F. Englert, A. Sevrin, W. Troost, A. Van Proeyen and P. Spindel, J. Math. Phys. 29, 281 (1988).

[4] I. Bena, Phys. Rev. D 62, 126006 (2000) [arXiv:hep-th/0004142].

[5] A. Basu and J. A. Harvey, Nucl. Phys. B 713, 136 (2005) [arXiv:hepth/0412310].

[6] D. S. Berman and N. B. Copland, Nucl. Phys. B 723, 117 (2005) [arXiv:hep-th/0504044]; Phys. Lett. B 639, 553 (2006) [arXiv:hepth/0605086]

[7] O. J. Ganor, Phys. Rev. D 75, 025002 (2007) [arXiv:hep-th/0609107].

[8] C. Papageorgakis and S. Ramgoolam, Int. J. Mod. Phys. A 21, 6055 (2006) [arXiv:hep-th/0603239].

[9] A. Gustavsson, arXiv:0709.1260 [hep-th].

[10] S. Okubo, "Introduction to Octonion and Other Non-Associative Algebras in Physics", Montroll Memorial Lecture Series in Mathematical Physics, CUP (2005) 
[11] L. Takhtajan, Commun. Math. Phys. 160, 295 (1994) [arXiv:hepth/9301111].

[12] B. Pioline, Phys. Rev. D 66, 025010 (2002) [arXiv:hep-th/0201257].

[13] J. H. Schwarz, JHEP 0411, 078 (2004) [arXiv:hep-th/0411077].

[14] J. Bagger and N. Lambert, in preparation. 